\title{
Importance of Substrate-Particle Repulsion for Protein-Templated Assembly of Metal Nanoparticles
}

Kathryn N. Guye', Hao Shen ${ }^{2,3}$, Muammer Y. Yaman ${ }^{1}$, Gerald Y. Liao', David Baker ${ }^{2,3,4}$, David S. Ginger $* 1,5$

Supporting Information 1 - Extinction and Zeta Potential Measurements of Gold Nanoparticles

\begin{tabular}{|c|c|c|c|c|}
\hline Diameter & $\lambda_{\max , \text { citrate }}$ & $\boldsymbol{\lambda}_{\max , \mathbf{C T A B}}$ & $\zeta_{\text {citrate }}$ & $\zeta_{\text {CTAB }}$ \\
\hline $10 \mathrm{~nm}$ & $518 \mathrm{~nm}$ & $524 \mathrm{~nm}$ & $-33 \mathrm{mV}$ & $+31 \mathrm{mV}$ \\
\hline $50 \mathrm{~nm}$ & $530 \mathrm{~nm}$ & $533 \mathrm{~nm}$ & $-45 \mathrm{mV}$ & $+42 \mathrm{mV}$ \\
\hline $100 \mathrm{~nm}$ & $574 \mathrm{~nm}$ & $577 \mathrm{~nm}$ & $-51 \mathrm{mV}$ & $+34 \mathrm{mV}$ \\
\hline
\end{tabular}

Table S1. Table showing the localized surface plasmon resonance (LSPR, $\lambda_{\max }$ ) and average zeta potential $(\zeta)$ for all nanoparticle solutions before and after the citrate-to-CTAB ligand exchange for each nanoparticle solution. A slight redshift in the LSPR is expected when the replacement ligand has a higher refractive index than the original ligand. ${ }^{1}$ The zeta potential of the nanoparticles changes from negative-to-positive with the ligand exchange, consistent with a citrate-to-CTAB ligand exchange. We note that zeta potential of the CTAB-capped nanoparticles do not trend with nanoparticle size. Therefore, increased repulsion due to an increasing zeta potential magnitude is not the cause of the reduced attachment of larger nanoparticles, rather the size-dependent substrateparticle repulsion determines attachment density.
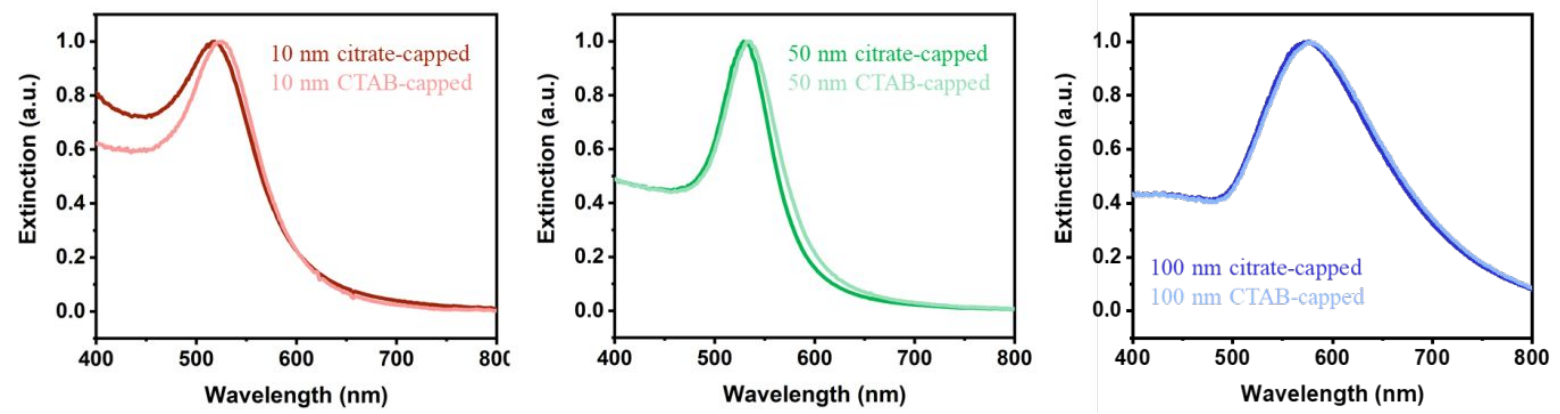

Figure S1. Normalized extinction plots of the $10 \mathrm{~nm}$ (red), $50 \mathrm{~nm}$ (green), and $100 \mathrm{~nm}$ (blue) gold nanoparticles as citrate-capped (darker traces) and after the citrate-to-CTAB ligand exchange (lighter traces). As discussed above, a slight redshift due to the CTAB capping is observed for each sample, however the line shape of the extinction traces do not drastically change, indicating nanoparticles remain monodisperse after ligand exchange. 


\section{Supporting Information 2 - Description of Intersurface Forces During Step 3 of the Assembly Process}

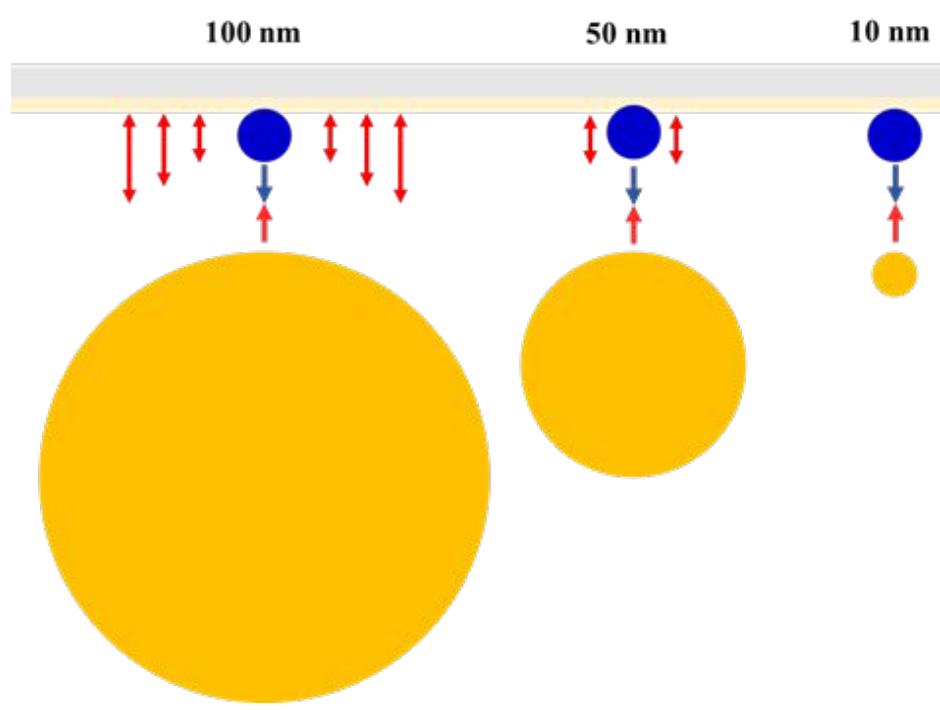

Figure S2. Free body diagram of the relative electrostatic forces acting on gold nanoparticles of varying sizes in the assembly geometry. There are three main elements to take into consideration in this system - the planar, positively-charged substrate; the cylindrical, negatively-charged protein nanofibers; and the spherical, positively-charged gold nanoparticles. Here, gold nanoparticles (yellow circles) approach a cross-section of the protein nanofiber (blue circles) anchored to the silane monolayer (beige plane) on the glass substrate (grey plane).

The protein-particle interaction is characterized as an attractive force (converging blue-red arrows) due to their opposite surface charges. The substrate-particle interaction, however, is characterized as an electrostatic repulsive force (diverging red-red arrows). While this repulsive force drives specific binding and prevents aggregation of the particles, it may also repel the particles from the substrate and therefore the protein nanofiber, preventing nanoparticle assembly.

In addition to the electrostatic forces, according to DLVO theory, ${ }^{2}$ all elements experience van der Waals attraction between them, which is presumed to be the driving force behind nonspecific attachment.

Furthermore, the nanoparticles in solution are subject to a downward gravitational force. By placing the protein-functionalized substrate face-down in the nanoparticle solution, gravitational sedimentation of gold nanoparticles does not contribute to the overall attachment of nanoparticles. 
Supporting Information 3 - Representative Images of Composites at pH 4, 7, and 10

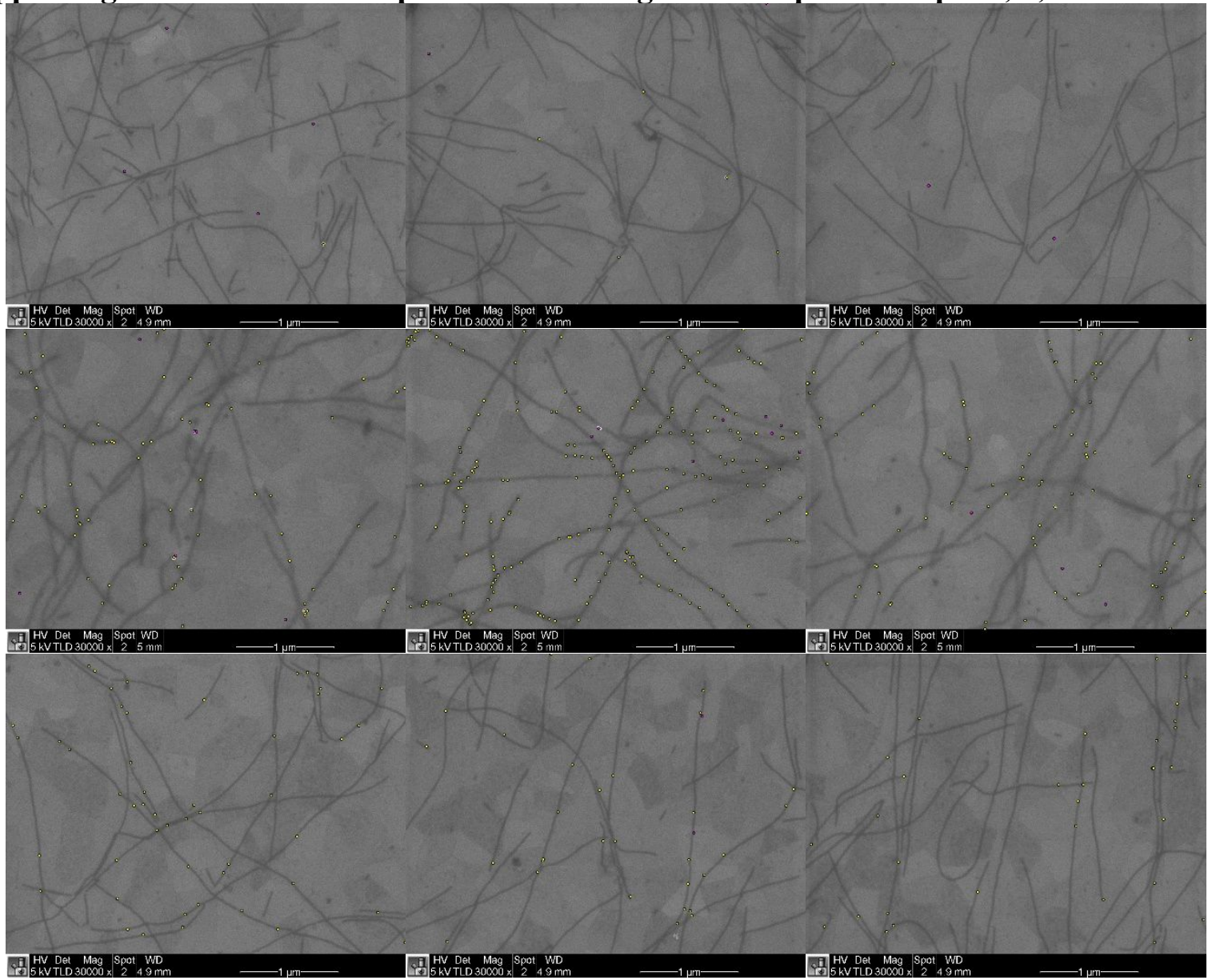

Figure S3a. Representative images from three samples of composites assembled at $\mathrm{pH} 4$. 


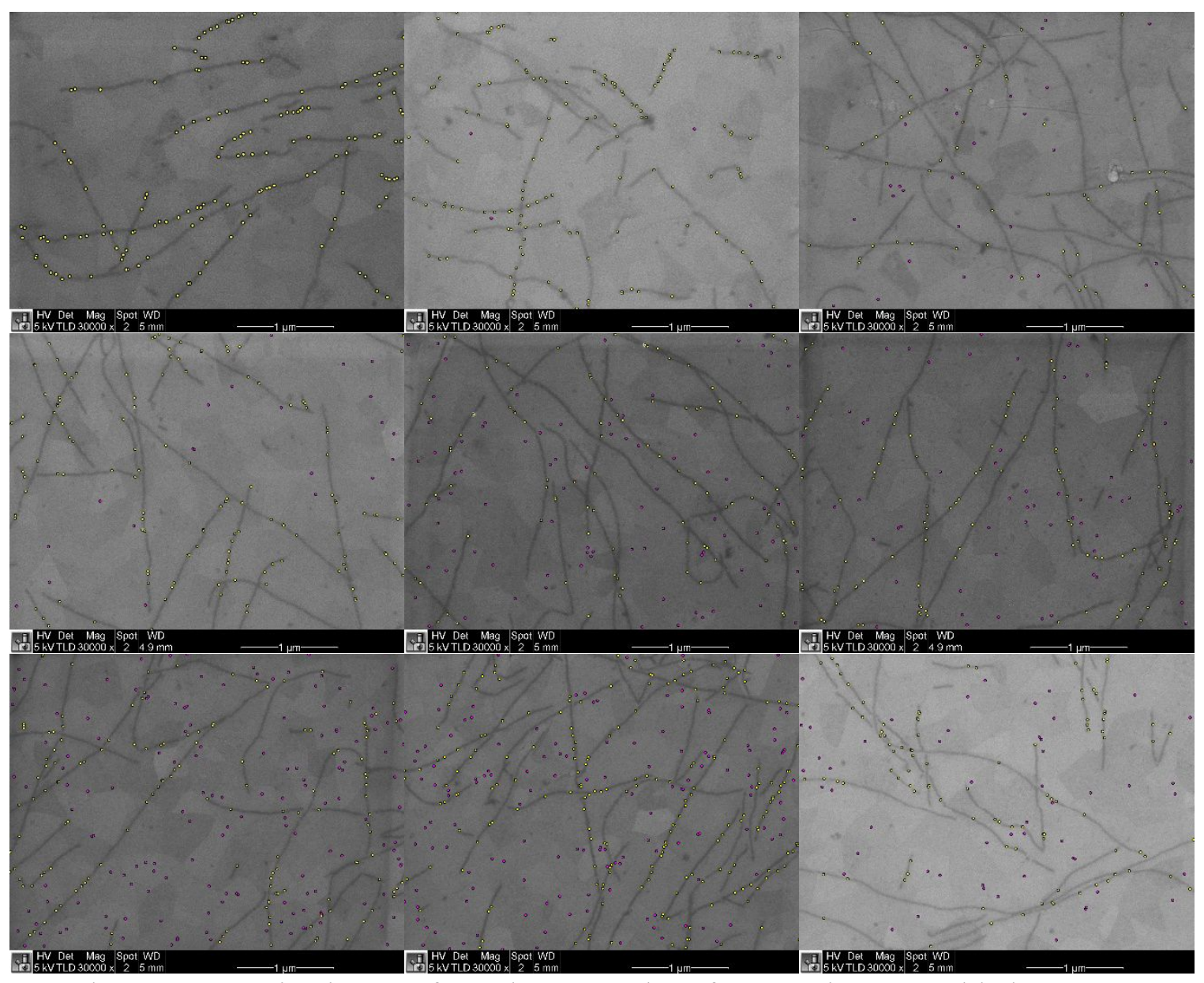

Figure S3b. Representative images from three samples of composites assembled at $\mathrm{pH} 7$. 




Figure S3c. Representative images from three samples of composites assembled at $\mathrm{pH} 10$. 


\section{Supporting Information 4 - Calculation of Theoretical Protonation of APTES and Protein Nanofibers as a Function of $\mathbf{p H}$}

For a monolayer of APTES, the $\mathrm{pK}_{\mathrm{a}}$ has been measured at 7.6. ${ }^{3}$ The ratio of protonated APTES, abbreviated as H:APTES(+) to neutral APTES was then calculated as:

$$
\frac{H: A P T E S(+)}{\text { Total APTES }}=1-\frac{\frac{K a}{H}}{\frac{K a}{H}+1}
$$

Unlike the silane monolayer, the protein nanofibers do not only go from charged-to-neutral, but rather negatively-charged, to neutral, to positively-charged as the protein behaves as a collection of $\mathrm{pH}$-responsive residues and therefore $\mathrm{pH}$-responsive functional groups. Therefore, rather than using a single functional group's $\mathrm{pK}_{\mathrm{a}}$, we calculated the isoelectric point ${ }^{4}$ of the protein to be 5.66. At a $\mathrm{pH}$ below the isoelectric point, the net charge of the protein is positive; at $\mathrm{pH} 5.66$, the average charge of the protein is assumed to be neutral; and above $\mathrm{pH} 5.66$, the protein is net negative. Heterogeneity of residue positioning, however, means electrostatic interactions with the nanoparticles can occur as soon as the carboxylic acid residues begin deprotonating. To visualize the fraction of negatively-charged residues to residues of all charge states, we calculated:

$$
\frac{\operatorname{Protein}(-)}{\text { Total Protein }}=\frac{\frac{K I}{H}}{\frac{K I}{H}+1}
$$

\section{Supporting Information 5 - Example of Particle Identification and Nearest Neighbors} Labeling
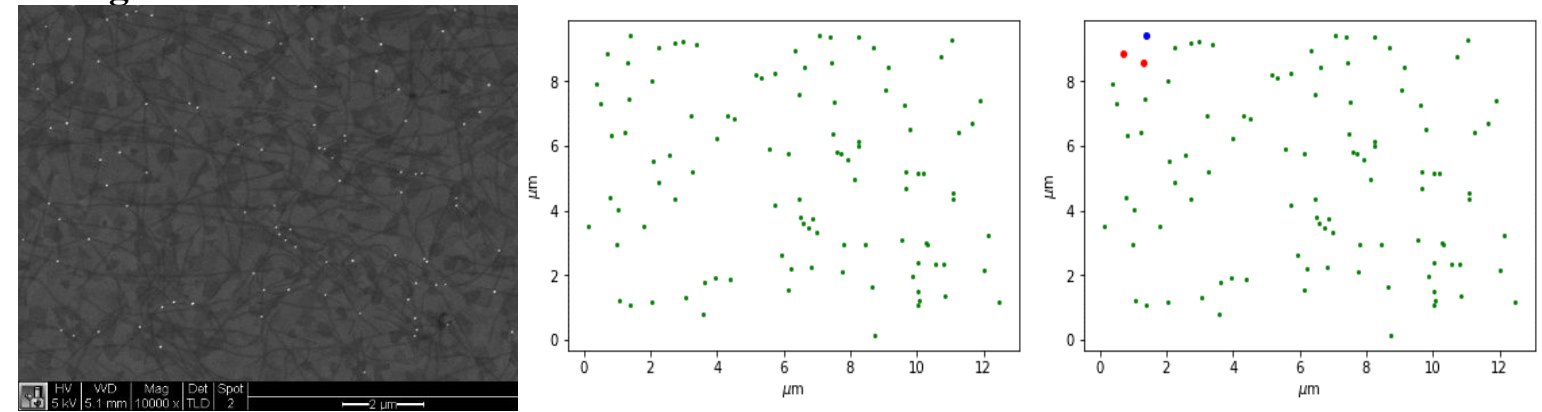

Figure S5. Nanoparticle labeling of a $50 \mathrm{~nm}$ gold nanoparticle composite sample for coordinate identification and nearest neighbors calculations. (left) SEM micrograph, (center) nanoparticle positions, (right) example of a nanoparticle (blue) and its labeled two nearest neighbors (red). 


\section{Supporting Information 6 - Variance of Nanoparticle Zeta Potential by pH}

\begin{tabular}{|c|c|}
\hline Diameter/pH & Average $\boldsymbol{\Delta} \boldsymbol{}$ \\
\hline $10 \mathrm{~nm} / \mathrm{pH} 4$ & $0.6 \mathrm{mV} / 0.4 \mathrm{mV}$ \\
\hline $10 \mathrm{~nm} / \mathrm{pH} 7$ & $1.8 \mathrm{mV} / 1.1 \mathrm{mV}$ \\
\hline $10 \mathrm{~nm} / \mathrm{pH} 10$ & $-1.2 \mathrm{mV} /-0.6 \mathrm{mV}$ \\
\hline $50 \mathrm{~nm} / \mathrm{pH} 5$ & $-5.8 \mathrm{mV}$ \\
\hline $50 \mathrm{~nm} / \mathrm{pH} 9$ & $-3.9 \mathrm{mV}$ \\
\hline
\end{tabular}

Figure S6. The change of zeta potential $(\Delta \zeta)$ of CTAB-capped gold nanoparticles by varying solution $\mathrm{pH}$ compared to the zeta potential of nanoparticles without buffer. Two solutions of separately-prepared $10 \mathrm{~nm}$ CTAB-capped gold nanoparticles were measured to show $\mathrm{pH}$ independent behavior is not solution-dependent. Although there is some slight zeta potential shift at different $\mathrm{pH}$ values, they do not track monotonically with $\mathrm{pH}$. While this may contribute to observed sample-to-sample variation, it is unlikely that $\mathrm{pH}$ affects the surface potential of nanoparticles significantly enough to contribute to the overall observed trends. This is consistent with $\mathrm{CTAB}$ containing a non-pH-responsive quaternary ammonium cation.

We note the change of zeta potential for the $50 \mathrm{~nm}$ CTAB-capped gold nanoparticles is slightly larger than observed for the $10 \mathrm{~nm}$ nanoparticles due to the addition of buffer. They are, however, similar to one another, which rules out nanoparticle surface potential as a contributing factor to the trends observed in Figure 4 in the main text.

\section{References}

1. Willets, K. A.; \& Van Duyne, R. P. Localized Surface Plasmon Resonance Spectroscopy and Sensing. Annu. Rev. Phys. Chem. 2007, 58, 267-297.

2. Israelachvili, J. N. Intermolecular and Surface Forces. (Elsevier Science \& Technology, 2010).

3. Zhang, H.; He, H. X.; Wang, J.; Mu, T.; \& Liu, Z. F. Force titration of amino groupterminated self-assembledmonolayers using chemical force microscopy. Appl. Phys. A Mater. Sci. Process. 1998, 66, 269-271.

4. Gasteiger, E.; Hoogland, C.; Gattiker, A.; Duvaud, S.; Wilkins, M. R.; Appel, R. D.; \& Bairoch, A. The Proteomics Protocols Handbook. Proteomics Protoc. Handb. 2005, 571608 doi:10.1385/1592598900. 